\title{
Presumed prenatal closure of foramen ovale and persistent pulmonary hypertension of the newborn
}

\section{Brief Report}

Cite this article: Stock K, Michel M, Schermer E, Ralser E, Kiechl-Kohlendorfer U, Geiger R, and Podnar T (2020) Presumed prenatal closure of foramen ovale and persistent pulmonary hypertension of the newborn. Cardiology in the Young 30: 281-283. doi: 10.1017/ S1047951119003044

Received: 5 August 2019

Revised: 18 September 2019

Accepted: 20 November 2019

First published online: 17 December 2019

\section{Keywords:}

Neonate; circulatory collapse; foramen ovale closure

\section{Author for correspondence:}

Tomaž Podnar, MD, PhD, Paediatrics III (Paediatric Cardiology, Pulmonology, Allergology and Cystic Fibrosis), Department of Child and Adolescent Health, Medical University of Innsbruck, Anichstrasse 35, 6020 Innsbruck, Austria. Tel: +43 51250480085 ; Fax: +43 512504 23484;

E-mail: tomaz.podnar@tirol-kliniken.at
(C) The Author(s) 2019. This is an Open Access article, distributed under the terms of the Creative Commons Attribution licence (http:// creativecommons.org/licenses/by/4.0/), which permits unrestricted re-use, distribution, and reproduction in any medium, provided the original work is properly cited.

\section{Katharina Stock ${ }^{1,2}$, Miriam Michel ${ }^{1}$, Elisabeth Schermer ${ }^{1}$, Elisabeth Ralser ${ }^{2}$, Ursula Kiechl-Kohlendorfer ${ }^{2}$, Ralf Geiger ${ }^{1}$ and Tomaž Podnar ${ }^{1}$ (1)}

${ }^{1}$ Paediatrics III (Paediatric Cardiology, Pulmonology, Allergology and Cystic Fibrosis), Department of Child and Adolescent Health, Medical University of Innsbruck, Innsbruck, Austria and 2Paediatrics II (Neonatology), Department of Child and Adolescent Health, Medical University of Innsbruck, Innsbruck, Austria

\section{Abstract}

Prenatal closure of foramen ovale without CHD is a rarely reported entity. Therefore, clinical and echocardiographic findings are poorly defined in these patients. We report a patient with prenatal closure of foramen ovale that presented with severe pulmonary hypertension of the newborn and left ventricular failure. Judicious management strategies were utilised to successfully treat both life-threatening conditions.

Prenatal restriction or closure of foramen ovale without CHD is associated with serious consequences. Recently, the incidence of restrictive and closed foramen ovale was reported to be 0.89 and $0.07 \%$, respectively. ${ }^{1}$ Both entities are often associated with persistent pulmonary hypertension of the newborn. Mortality may be higher in patients with closed foramen ovale. ${ }^{1,2}$ Echocardiographic findings are well defined in patients with restrictive foramen ovale. ${ }^{2}$ However, due to a small number of reported patients, clinical and echocardiographic findings are less clearly defined in patients with closed foramen ovale. ${ }^{1,2}$ Furthermore, the left heart function was not clearly evaluated in these patients. Therefore, we report a patient who was diagnosed immediately after birth with closed foramen ovale, severe persistent pulmonary hypertension of the newborn, and profound left heart failure.

\section{Case}

A male infant was born at term with a birth weight of $3090 \mathrm{~g}$. The prenatal course, delivery and immediate postnatal course were normal. In retrospect, we recognised that differential cyanosis was present immediately after birth (Fig 1). Forty minutes after birth the newborn developed severe respiratory distress and profound central cyanosis. Pre-ductal percutaneous oxygen saturation was $60 \%$ and was significantly lower post-ductal. Venous blood gas analysis showed pH 6.98, pCO2 $82.9 \mathrm{mmHg}$, lactate $83 \mathrm{mg} / \mathrm{dl}$, and BE-18.6 mmol/L. The newborn was immediately intubated. Despite $\mathrm{FiO} 2$ of $100 \%$ and mechanical ventilation, the pre-ductal percutaneous oxygen saturation remained low. CHD was suspected and therefore prostaglandin infusion was started. Chest X-ray revealed lung oedema. Transthoracic echocardiography excluded CHD. However, a compact interatrial septum with completely closed foramen ovale was noted (Fig 2). There was a prominent right heart and signs of persistent pulmonary hypertension with right to left shunt across the widely open ductus arteriosus. In addition, the free wall of the left ventricle was completely immobile and significant mitral regurgitation was present (Supplementary Video S1). The left heart structures were smaller but not hypoplastic. Oscillatory and conventional ventilation with high positive inspiratory pressure and $\mathrm{FiO} 2$ of $100 \%$ was needed. Therapy with $20 \mathrm{ppm}$ iNO, continuous deep sedation, analgesia, and relaxation were initiated. Systemic perfusion was duct-dependent, and therefore prostaglandin infusion was continued. Due to severe left heart failure and lung oedema, inotropic support was initiated and diuretics were administered. Initially, dopamine, adrenaline, and noradrenaline infusions were given simultaneously. In addition, levosimendan infusion was given. After stabilisation, we continued only with milrinone infusion. Left ventricular function and lactate level normalised within 48 hours, and inotropic support was gradually discontinued (Supplementary Video S2). Thereafter, pulmonary hypertension therapy was intensified. After initiation of bosentan therapy $(2 \mathrm{mg} / \mathrm{kg}$ by stomach probe $/ 12 \mathrm{~h}$ ) significant echocardiographic improvement was observed. However, pulmonary hypertension was still relevant and therefore continuous intravenous sildenafil infusion was added $(0.4 \mathrm{mg} / \mathrm{kg}$ i.v. loading administered over 3 hours, followed by $0.067 \mathrm{mg} / \mathrm{kg} / \mathrm{h}$ infusion) contributing to normalisation of pulmonary pressures and clinical improvement. After complete reversion of ductal shunting (i.e., from right to left into left to right) prostaglandin infusion was discontinued. 


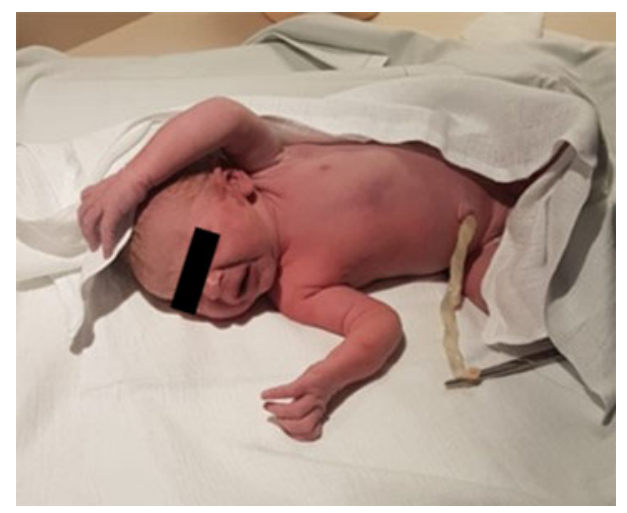

Figure 1. Differential cyanosis. The patient presented in this report. Only the upper right extremity is pink, while the remaining body is cyanotic.



Figure 2. Atrial septum. Subcostal view depicting thick closed interatrial septum. $\mathrm{IAS}=$ interatrial septum; LA = left atrium; RA = right atrium

Inhalation of NO was tapered gradually and after 12 days the patient was successfully extubated. The patient was discharged with oral sildenafil and bosentan therapy. Follow-up MRI evaluation excluded hypoxic-ischemic encephalopathy. The neurological examination showed no abnormalities and the child's development is entirely normal. At the age of 9 months sildenafil and bosentan were discontinued. We continue to follow the patient.

\section{Discussion}

Data on clinical and echocardiographic findings in patients with closed foramen ovale without CHD are scarce. Hagen et al reported two patients diagnosed prenatally with closed foramen ovale. ${ }^{3}$ Both were recognised due to enlarged right heart dimensions while left heart size was normal in both of them. Because of a progression of right ventricular dilation caesarean section was performed near term and the right ventricular dimensions normalised after birth in both patients.

Uzun et al reported a series of 23 patients with restrictive foramen ovale. ${ }^{2}$ All of them were recognised prenatally due to right heart dilatation. Four of their patients also had impaired left ventricular function. In two, left ventricular function normalised after birth following treatment. In a patient with associated Alström syndrome, left ventricular dilation persisted. The fourth patient had VACTERL association and presented with impairment of both, left and right ventricular function. This patient was born very preterm, had severe persistent pulmonary hypertension of the newborn and died soon after birth.

$\mathrm{Gu}$ et al reported a series of 60 foetuses with restrictive foramen ovale and 5 with closed foramen ovale. ${ }^{1}$ In one of the foetuses with closed foramen ovale, authors observed decreased systolic function of both ventricles and hydrops. The patient was delivered very preterm and died after birth.

$\mathrm{Li}$ et al reported two patients with premature closure or restriction of the foramen ovale both diagnosed during the third trimester of pregnancy. ${ }^{4}$ In both foetuses, it was difficult to distinguish between closure and restriction of foramen ovale using conventional two-dimensional and colour Doppler imaging.

In contrast to the reported patients, our patient was diagnosed after birth. Echocardiography revealed a thick completely closed atrial septum. In addition to signs of persistent pulmonary hypertension of the newborn, the most prominent echocardiographic finding was a severe left ventricular impairment. Systemic circulation was duct-dependent despite normal-sized left heart structures. At the beginning, we managed the circulation as in a patient having a hypoplastic left heart syndrome, that is, a single-ventricle circulation with a duct-dependent systemic circulation (Supplementary material - Management suggestions). Therefore, prostaglandin infusion was continued. Measures to prevent pulmonary hypertensive crisis were imperative and myocardial function was supported by inotropes. At that stage, pulmonary hypertension was managed with $\mathrm{FiO} 2$ of $100 \%$ and $20 \mathrm{ppm}$ iNO. Circulatory collapse and severe left ventricular dysfunction were managed by simultaneous infusions of dopamine, adrenaline, and noradrenaline. To further improve myocardial function, levosimendan infusion without bolus was added. After stabilisation of the circulation and improvement of the left ventricular function, only milrinone infusion was given. Left ventricular impairment resolved completely within 48 hours and from that time on we managed the haemodynamics as in a biventricular circulation with severe persistent pulmonary hypertension of the newborn. At that point, we intensified treatment of pulmonary hypertension first with bosentan administration by stomach probe and later with addition of sildenafil infusion. Due to progressive improvement as reflected also by gradual normalisation of lactate level, we continued to manage the patient without extracorporeal membrane oxygenation support.

Late clinical manifestations of mitral valve disease and severe pulmonary hypertension were reported in a patient diagnosed in utero with premature closure of foramen ovale. ${ }^{5}$ Therefore, we continue to follow our patient.

Left ventricular dysfunction was a prominent component of the clinical picture after birth. We speculate that inadequate left ventricular preload due to the closure of the foramen ovale and severe pulmonary vascular resistance in association with severe acidosis caused stunned myocardium. In this setting, aggressive management of persistent pulmonary hypertension before normalisation of the left ventricular function could worsen lung oedema and haemodynamic instability.

Supplementary Material. To view supplementary material for this article, please visit https://doi.org/10.1017/S1047951119003044

Acknowledgements. We would like to acknowledge the patient's parents for providing Figure 1.

Financial Support. This research received no specific grant from any funding agency, commercial or not-for-profit sectors. 
Conflicts of Interest. None.

Ethical Standards. The authors assert that all work reported complies with the ethical standards of the Helsinki convention, and institutional and national research committee.

\section{References}

1. Gu X, Zhang Y, Han J, et al. Isolated premature restriction or closure of foramen ovale in fetuses: echocardiographic characteristics and outcome. Echocardiography. 2018; 35: 1189-1195.
2. Uzun O, Babaoglu K, Ayhan YI, et al. Diagnostic ultrasound features and outcome of restrictive foramen ovale in fetuses with structurally normal hearts. Pediatr Cardiol. 2014; 35: 943-952.

3. Hagen A, Albig M, Schmitz L, et al. Prenatal diagnosis of isolated foramen ovale obstruction. A report of two cases. Fetal Diagn Ther. 2005; 20: 70-73.

4. Li YD, Li ZA, He YH. Premature closure or restriction of the foramen ovale. Prenatal diagnosis by directional enhanced flow imaging. J Ultrasound Med 2013; 32: 1291-1294.

5. Iwamoto $\mathrm{Y}$, Tamai A, Kawasaki H, et al. Late clinical manifestations of mitral valve disease and severe pulmonary hypertension in a patient diagnosed with premature closure of foramen ovale during fetal life. World J Pediatr 2011; 7: $182-184$. 